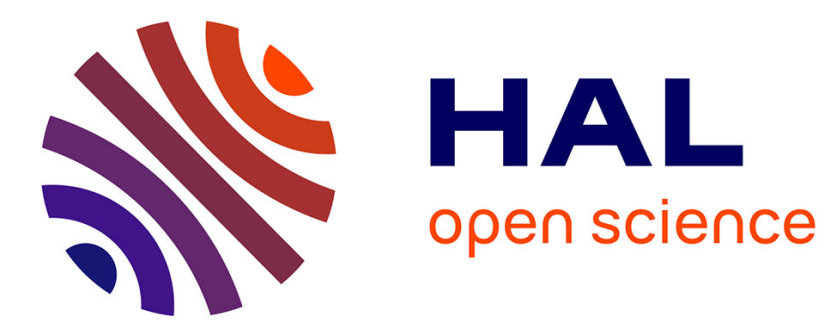

\title{
Interval state estimation for uncertain nonlinear systems
} Gang Zheng, Denis Efimov, Wilfrid Perruquetti

\section{To cite this version:}

Gang Zheng, Denis Efimov, Wilfrid Perruquetti. Interval state estimation for uncertain nonlinear systems. IFAC Nolcos 2013, Sep 2013, Toulouse, France. hal-00844397

\section{HAL Id: hal-00844397 https://hal.inria.fr/hal-00844397}

Submitted on 15 Jul 2013

HAL is a multi-disciplinary open access archive for the deposit and dissemination of scientific research documents, whether they are published or not. The documents may come from teaching and research institutions in France or abroad, or from public or private research centers.
L'archive ouverte pluridisciplinaire HAL, est destinée au dépôt et à la diffusion de documents scientifiques de niveau recherche, publiés ou non, émanant des établissements d'enseignement et de recherche français ou étrangers, des laboratoires publics ou privés. 


\title{
Interval state estimation for uncertain nonlinear systems
}

\author{
Gang Zheng * Denis Efimov*,** Wilfrid Perruquetti *
* Non-A team of INRIA-LNE, Parc Scientifique de la Haute Borne, 40 avenue Halley, Bt.A Park Plaza, 59650 Villeneuve d'Ascq, France, $\{$ Denis.Efimov; Wilfrid.Perruquetti;Gang.Zheng\}@inria.fr ** Department of Control Systems and Informatics, Saint Petersburg State University of Information Technologies Mechanics and Optics \\ (ITMO), Kronverkskiy av. 49, Saint Petersburg, 197101, Russia.
}

\begin{abstract}
The objective of this work is to develop some design methods of interval observers for a class of nonlinear continuous-time systems. It is assumed that the estimated system can be represented as a superposition of the nominal subsystem (belonged to the class of uniformly observable systems) and a Lipschitz nonlinear perturbation vanishing at the origin. Then it is shown there exists an interval observer for the system that estimates the set of admissible values for the state consistent with the output measurements. An example of the observer application is given with computer simulation results.
\end{abstract}

\section{INTRODUCTION}

The state estimation problem of uncertain nonlinear systems is studied in this work. In particular we are interested in the case when the model is nonlinear parameterized by a vector of unknown parameters $\theta$ and the model equations do not belong to a canonical form. Usually in such a case it is necessary to apply a transformation of coordinates representing the system in a canonical form with posterior design of an observer Besançon [2007], Nijmeijer and Fossen [1999]. The presence of unknown parameters may seriously complicate the design of a required transformation of coordinates, since the transformation has to be dependent on $\theta$. In this case the initial problem of the state estimation can be replaced with a relaxed one dealing with approximation of the interval of admissible values of the state vector.

In the sequel, only single output systems are considered. Suppose that the unknown (may be time-varying) parameters $\theta$ belong to a compact set $\Theta \subset \mathbb{R}^{p}$, then the plant dynamics under consideration is given by

$$
\begin{aligned}
& \dot{x}=f^{\theta}(x)+B^{\theta}(x) u, \\
& y=h^{\theta}(x),
\end{aligned}
$$

where $x$ belongs to an open subset $\Omega$ of $\mathbb{R}^{n}$ and the initial state value belongs to a compact set $I_{0}\left(x_{0}\right)=\left[\underline{x}_{0}, \bar{x}_{0}\right]$; $y \in \mathbb{R}$ and $u \in \mathbb{R}^{m}$ represent respectively the output and the input. $B^{\theta}(x)$ is the input gain matrix whose column vectors are $b_{i}^{\theta}, i=1, \ldots, m$. The vector fields $f^{\theta}$ and $b_{i}^{\theta}$, and the output function $h^{\theta}$ are parameterized by $\theta \in \Theta$.

\footnotetext{
* This work was supported by EU Interreg IV A 2 Mers Seas Zeeën Cross-border Cooperation Programme under SYSIASS project 06020. It was also supported by Ministry of Higher Education and Research, Nord-Pas de Calais Regional Council and FEDER through the "Contrat de Projets Etat Region (CPER) CIA 2007-2013".
}

Assumptions A1): For all $x \in \Omega \subset \mathbb{R}^{n}$ and all $\theta \in \Theta$ we assume that

$$
\begin{aligned}
f^{\theta}(x) & =f(x)+\delta f(x, \theta), \\
b_{i}^{\theta}(x) & =b_{i}(x, \theta), \\
h^{\theta}(x) & =h(x)+\delta h(x, \theta)
\end{aligned}
$$

for some known nominal functions $f$ and $h$, where $\delta f$ and $b_{i}$ are assumed to be at least continuous and such that (for all $\theta \in \Theta)$

$$
\begin{gathered}
f(0)=0, \delta f(0, \theta)=0, \\
b_{i}^{\theta}(0)=0, \delta h(0, \theta)=0 .
\end{gathered}
$$

Thus system (1)-(2) with (3)-(5) reads as:

$$
\left\{\begin{array}{l}
\dot{x}=f(x)+B(x, \theta) u+\delta f(x, \theta), \\
y=h(x)+\delta h(x, \theta) .
\end{array}\right.
$$

Despite of the existence of many solutions for observer design Besançon [2007], Nijmeijer and Fossen [1999], a design of state estimators for (8) is rather complicated since the system is intrinsically nonlinear and it has uncertain terms in the state and the output equations. The idea is to relax the estimation goal making an evaluation of the interval of admissible values for the state applying the theory of set-membership or interval estimation Gouzé et al. [2000], Mazenc and Bernard [2010], Walter et al. [1996]. Contrarily the conventional case, where a pointwise value of the state is the objective for estimation, in the interval estimation two bounds on the set of admissible values are calculated and the width of the estimated interval is dependent on the model uncertainty.

Recently the interval observers have been proposed for a special class of nonlinear systems Raïssi et al. [2012], the model (1)-(2) is a generalization of that case. Applying a coordinate transformation to a canonical form computed for the known nominal system, we are going to estimate 
the interval value of the state of the uncertain system (8) improving the result from Raïssi et al. [2012]. Another solution has been presented in Meslem and Ramdani [2011], where a hybrid interval observer design is presented for a class of continuous-time nonlinear systems. In the present work we are going to avoid the complexity of the hybrid systems framework developing a continuoustime interval observer. For upper-triangular systems, an iterative design procedure for robust interval observers is proposed in Mazenc and Bernard [2012], which is started from the assumption that for each subsystem a robust interval observer has been designed. The result of this work can be considered as a complementary method for such an observer syntheses for a nonlinear system.

The outline of this paper is as follows. Some preliminary results and notations are given in Section 2. The precise problem formulation is presented in Section 3. The main results are described in Section 4. An example of computer simulation is given in Section 5 .

\section{PRELIMINARIES}

\subsection{Notations}

- $\mathbb{R}$ denotes the set of real numbers and $\mathbb{R}_{+}=\{x \in \mathbb{R}$ : $x \geq 0\}$.

- $L_{f} h(x)=\frac{\partial}{\partial x} h(x) \cdot f(x)$ denotes the Lie derivative of $h$ along the vector field $f$, and $L_{f}^{n} h=L_{f}\left(L_{f}^{n-1} h\right)$ is the $n$-th Lie derivative of $h$ along the vector field $f$.

- $a \mathcal{R} b$, element-wise relation $\mathcal{R}$ ( $a$ and $b$ are vectors or matrices): for example $a<b$ (vectors) means $\forall i: a_{i}<b_{i}$

- for a matrix $P=P^{T}$, the relation $P \preceq 0$ means that the matrix is negative semidefinite.

- for a matrix $A \in \mathbb{R}^{n}$ define $A^{+}=\max \{0, A\}$ and $A^{-}=A^{+}-A$.

- for a matrix (function) $A$ the symbol $A_{i}$ denotes its $i^{\text {th }}$ column, for a vector (function) $b$ the symbol $b_{i}$ denotes its corresponding element.

- a matrix $A \in \mathbb{R}^{n \times n}$ is called Metzler if all its elements outside the main diagonal are nonnegative.

- a Lebesgue measurable function $u: \mathbb{R}_{+} \rightarrow \mathbb{R}^{m}$ belongs to the space $\mathcal{L}_{\infty}$ if $\|u(t)\|<+\infty$ for almost every $t \in \mathbb{R}_{+}$.

\subsection{Backgrounds on cooperative/comparison systems}

The notions of Comparison systems and Cooperative systems have appeared separately, but they concern the same class of systems:

- Comparison systems: when dealing with a qualitative property involving solutions of a complex system, it is sometimes of interest to obtain a simpler system whose solutions overvalue the solutions of the initial system in some sense. For ODE, the contributions of Müller [1926], Kamke [1932], Wazewski [1950] are probably the most important in this field: they give necessary and sufficient hypotheses ensuring that the solution of $\dot{x}=f(t, x)$, with initial state $x_{0}$ at time $t_{0}$ and function $f$ satisfying the inequality $f(t, x) \leq g(t, x)$ is overvalued by the solution of the so-called "comparison system" $\dot{z}=g(t, z)$, with initial state $z_{0} \geq x_{0}$ at time $t_{0}$, or, in other words, conditions on function $g$ that ensure $x(t) \leq z(t)$ for $t \geq t_{0}$. These results were extended to many different classes of dynamical systems (Bitsoris [1978], Dambrine [1994], Dambrine et al. [1995], Dambrine and Richard [1993, 1994], Grujic̀ et al. [1987], Laksmikantham and Leela [1969], Matrosov [1971], Perruquetti et al. [1995a,b], Tokumaru et al. [1975]).

- Cooperative systems: this class of systems includes those involving in $\mathbb{R}^{n}$ preserving positive order relation on initial data and input signals Smith [1995], i.e. if the initial conditions and properly rescaled inputs are positive, then so is the corresponding solution. According to the above explanations, if $\dot{z}=g(t, z)$ is a comparison system for $\dot{x}=f(t, x)$, then the error $e(t)=z(t)-x(t)$ behavior is cooperative.

From these results one can deduce the following corollary: Corollary 1. Assume that:

H1) $A$ is a Metzler matrix,

H2) $b(t) \in \mathbb{R}_{+}^{n}, \forall t \geq t_{0} \in \mathbb{R}_{+}$,

H3) the exogenous signal $b$ is sufficiently smooth such that the system

$$
\frac{d x(t)}{d t}=A x+b(t)
$$

possesses, for every $x\left(t_{0}\right) \in \mathbb{R}_{+}^{n}$, a unique solution $x(t)$ for all $t \geq t_{0}$.

Then, for any $x\left(t_{0}\right) \in \mathbb{R}_{+}^{n}$, the inequality

$$
x(t) \geq 0
$$

holds for every $t \geq t_{0}$.

In other word, under conditions of Corollary $1, \mathbb{R}_{+}^{n}$ is positively invariant w.r.t (9).

\subsection{Canonical representation of a nonlinear system}

From (8), one obtains the nominal drift-system by setting $u=0, \delta f=0, \delta h=0$ in $(8)$ :

$$
\left\{\begin{array}{l}
\dot{x}=f(x) \\
y=h(x)
\end{array}\right.
$$

For a nonlinear system, "observability" depends on the considered state (local property) and control: this is the main reason why many different concepts related to observability exists Besançon [2007], Nijmeijer and Fossen [1999]. One of these is independent of the input and is called uniform observability.

Definition 2. The system (10) is said to be uniformly observable on $\Omega$ iff (if and only if) the function:

$$
\begin{aligned}
\Phi_{(10)} & : \mathbb{R}^{n} \rightarrow \mathbb{R}^{n} \\
x & \mapsto\left(h(x), L_{f} h(x), \ldots, L_{f}^{n-1} h(x)\right)^{T}
\end{aligned}
$$

defines a diffeomorphism from $\Omega$ onto $\Phi_{(10)}(\Omega)$.

From this definition, it follows that, on $\Omega$, in the global coordinates defined by $\zeta=\Phi_{(10)}(x)$, the system (10) can be rewritten as:

$$
\left\{\begin{array}{l}
\dot{\zeta}=\tilde{A} \zeta+\tilde{b} \varphi(\zeta) \\
y=x_{1}=\zeta_{1}
\end{array}\right.
$$

where 


$$
\begin{aligned}
\tilde{A} & =\left(\begin{array}{cccc}
0 & 1 & & 0 \\
& \ddots & \ddots & \\
& & \ddots & 1 \\
0 & & & 0
\end{array}\right), \\
\tilde{b} & =(0, \ldots, 0,1)^{T}, \\
\varphi(\zeta) & =L_{f}^{n} h(x) .
\end{aligned}
$$

The uniform observability is associated to the observability normal-form theorem given by Gauthier et al. [1992] and recalled hereafter.

Theorem 3. Assume that $\varphi: \Omega \rightarrow \mathbb{R}$ in the equation (12) can be extended to $\mathbb{R}^{n}$ by a globally-Lipschitz analytic function, then the system

$$
\left\{\begin{array}{l}
\dot{x}=f(x)+B(x) u \\
y=h(x)
\end{array}\right.
$$

is uniformly observable for any input iff it is diffeomorphic to a system of the form:

$$
\left\{\begin{array}{l}
\dot{\zeta}_{1}=\zeta_{2}+\sum_{j=1}^{m} g_{1, j}\left(\zeta_{1}\right) u_{j}, \\
\dot{\zeta}_{2}=\zeta_{3}+\sum_{j=1}^{m} g_{2, j}\left(\zeta_{1}, \zeta_{2}\right) u_{j}, \\
\vdots \\
\dot{\zeta}_{n-1}=\zeta_{n}+\sum_{j=1}^{m} g_{n-1, j}\left(\zeta_{1} \ldots \zeta_{n-1}\right) u_{j}, \\
\dot{\zeta}_{n}=\varphi(\zeta)+\sum_{j=1}^{m} g_{n, j}(\zeta) u_{j}, \\
y=\zeta_{1}=\tilde{C} \zeta
\end{array}\right.
$$

where $\tilde{C}=\left(\begin{array}{ll}1 & 0 \ldots 0\end{array}\right)$.

If we assume that $\varphi$ and $g_{i, j}$ verify $\varphi(0)=0, g_{i, j}(0, \ldots, 0)=$ 0 for $i=1, \ldots, n, j=1, \ldots, m$, then one can design a high gain observer (see Gauthier et al. [1992]). The forthcoming analysis is based on these results.

\section{PROBLEM FORMULATION}

The objective of this work is to design an interval observer for the system (1) under Assumption A1), i.e. for the system (8). We will not even assume that (8) is observable, but to use the results of the previous section, i.e. we will impose observability for the nominal system (10) only.

Assumptions A2): The nominal system (10) is uniformly observable.

Thus (8) may be not uniformly observable, however using a transformation of coordinates obtained for the nominal observable (under Assumption A2)) system (10), the system (8) can be transformed into the following one:

$$
\left\{\begin{array}{l}
\dot{\zeta}=\tilde{A} \zeta+\tilde{F}(\zeta, \theta)+\tilde{G}(\zeta, \theta) u \\
y=\tilde{C} \zeta+\tilde{H}(\zeta, \theta)
\end{array}\right.
$$

where $\tilde{G}(\zeta, \theta)$ has a triangular structure as $g$ in $(17)$ and

$$
\begin{aligned}
\tilde{F}(\zeta, \theta) & =\left(\tilde{F}_{1}(x, \theta), \ldots, \tilde{F}_{n}(x, \theta)\right)_{x=\Phi_{(10)}^{-1}(\zeta)}^{T}, \\
\tilde{F}_{i}(x, \theta) & =L_{\delta f(x, \theta)} L_{f(x)}^{i-1} h(x), i=1 \ldots n, \\
\tilde{H}(\zeta, \theta) & =\left.\delta h(x, \theta)\right|_{x=\Phi_{(10)}^{-1}(\zeta)} .
\end{aligned}
$$

To proceed we need the following assumption.

Assumptions A3): There exist a matrix $\tilde{L}$ and an invertible matrix $P$ such that the matrix $\tilde{A}-\tilde{L} \tilde{C}$ is similar to a Metzler matrix $A-L C$, where $A=P \tilde{A} P^{-1}, L=P \tilde{L}$, $C=\tilde{C} P^{-1}$.

The conditions of such a transformation matrix $P$ existence can be found in Raïssi et al. [2012], they are related with solution of a Sylvester equation. Since the pair $(\tilde{A}, \tilde{C})$ is observable, always there is a gain $\tilde{L}$ such that Assumption A3) is satisfied Raïssi et al. [2012]. Introducing the new coordinates $z=P \zeta$ we arrive at the desired representation of our system (1):

$$
\left\{\begin{array}{l}
\dot{z}=A z+F(z, \theta)+G(z, \theta) u \\
y=C z+H(z, \theta)
\end{array}\right.
$$

where the matrices $A, C$ are given in Assumption A3), and $H(z, \theta)=\tilde{H}\left(P^{-1} z, \theta\right), F(z, \theta)=P \tilde{F}\left(P^{-1} z, \theta\right), G(z, \theta)=$ $P \tilde{G}\left(P^{-1} z, \theta\right)$.

Remark 4. Since the origin of (8) is assumed to be an equilibrium (see (6) and (7)) and $\Phi_{(10)}$ is a diffeomorphism, we have

$$
G_{i, j}(0, \theta)=0, F(0, \theta)=0 .
$$

Let us remind that, since the initial condition $x_{0}$ for (1) is only known within a certain interval $I\left(x_{0}\right)=\left[\underline{x}_{0}, \bar{x}_{0}\right]$, then using the diffeomorphism $\Phi_{(10)}(x)$, the initial condition $z_{0}=P \Phi_{(10)}\left(x_{0}\right)$ is also known within a certain interval $I\left(z_{0}\right)=\left[\underline{z}_{0}, \bar{z}_{0}\right]$. Thus our original problem turns out to built two dynamical systems with the input $(u, y)$ and the outputs $\underline{z}(t)$ and $\bar{z}(t)$ such that for all $t \geq 0$ we have:

$$
\underline{z}(t) \leq z(t) \leq \bar{z}(t)
$$

\section{MAIN RESULTS}

\subsection{Bounding functions}

Since $\Theta$ is a compact set and by continuity of $F(z, \theta), H(z, \theta)$ and $G(x, \theta)$ (the functions $\delta f(x, \theta), b_{i}(x, \theta)$ and $\delta h(x, \theta)$ were assumed to be continuous and $\Phi$ given by (11) is a diffeomorphism), the elementwise minimum and maximum of $F(z, \theta), H(z, \theta)$ and $G(z, \theta) u$ (for a given $u$ ) in the domain $\theta \in \Theta, \underline{z} \leq z \leq \bar{z}$ exist. In order to built the observers, we need a more precise knowledge on these max and min functions. For this we need the following lemma. Lemma 5. Let $A \in \mathbb{R}^{n \times n}$, then by the definition $A=A^{+}-$ $A^{-}$and for any $[\underline{z}, \bar{z}] \subset \mathbb{R}^{n}$ and $z \in \mathbb{R}^{n}$ we have

$$
[\underline{z} \leq z \leq \bar{z}] \Longrightarrow A^{+} \underline{z}-A^{-} \bar{z} \leq A z \leq A^{+} \bar{z}-A^{-} \underline{z} .
$$

Lemma 6 . Let $\underline{A} \leq A \leq \bar{A}$ for some $\underline{A}, \bar{A}, A \in \mathbb{R}^{n \times n}$ and $\underline{x} \leq x \leq \bar{x}$ for $\underline{x}, \bar{x}, x \in \mathbb{R}^{n}$, then

$$
\begin{gathered}
\underline{A}^{+} \underline{x}^{+}-\bar{A}^{+} \underline{x}^{-}-\underline{A}^{-} \bar{x}^{+}+\bar{A}^{-} \bar{x}^{-} \leq A x \\
\leq \bar{A}^{+} \bar{x}^{+}-\underline{A}^{+} \bar{x}^{-}-\bar{A}^{-} \underline{x}^{+}+\underline{A}^{-} \underline{x}^{-} .
\end{gathered}
$$


All proofs are skipped due to the space limitation.

To apply these lemmas we have to introduce the following standard assumption in the estimation theory on boundedness of the state $x$ and the input $u$ values.

Assumptions A4): $x(t) \in \mathcal{X}$ and $u(t) \in \mathcal{U}$ for all $t \geq 0$, where $\mathcal{X} \subset \Omega$ and $\mathcal{U} \subset \mathbb{R}^{m}$ are two given compacts.

Under this assumption there exists a compact set $\mathcal{Z} \subset \mathbb{R}^{n}$ such that $z(t) \in \mathcal{Z}$ for all $t \geq 0$.

Lemma 7. There exist two functions $\bar{F}, \underline{F}: \mathbb{R}^{2 n} \rightarrow \mathbb{R}^{n}$ such that the following inequalities hold for all $\theta \in \Theta$ and $\underline{z} \leq z \leq \bar{z}$ :

$$
\underline{F}(\underline{z}, \bar{z}) \leq F(z, \theta) \leq \bar{F}(\underline{z}, \bar{z})
$$

and for a given submultiplicative norm $\|\cdot\|$

$$
\begin{aligned}
& \|\bar{F}(\underline{z}, \bar{z})-F(z, \theta)\| \leq \bar{l}_{\bar{F}}\|\bar{z}-z\|+\underline{l}_{\bar{F}}\|\underline{z}-z\|+l_{\bar{F}}, \\
& \|\underline{F}(\underline{z}, \bar{z})-F(z, \theta)\| \leq \bar{l}_{\underline{F}}\|\bar{z}-z\|+\underline{l}_{F}\|\underline{z}-z\|+l_{\underline{F}},
\end{aligned}
$$

for some positive constants $\bar{l}_{\bar{F}}, \underline{l}_{\bar{F}}, l_{\bar{F}}, \bar{l}_{\underline{F}}, \underline{l}_{\underline{F}}, l_{\underline{F}}$.

Remark 8. Thus the difference of functions $\bar{F}, \underline{F}$ and $F$ has a linear growth with respect to the interval width estimates $\underline{z}-z$ and $\bar{z}-z$.

Note that the values of constants $\bar{l}_{\bar{F}}, \underline{l}_{\bar{F}}, l_{\bar{F}}, \bar{l}_{\underline{F}}, \underline{l}_{\underline{F}}, l_{F}$ and functions $\underline{F}, \bar{F}$ are the worst case upper bounds. The goal of the lemma is to show that they exist and to provide some approximate outer estimates for them. For a particular application more accurate values may be computed.

Using Lemma 7, a similar result can be established for $H$, i.e. there exist two functions $\bar{H}, \underline{H}: \mathbb{R}^{2 n} \rightarrow \mathbb{R}^{n}$ such that the following inequalities holds for all $\theta \in \Theta$ and $\underline{z} \leq z \leq \bar{z}$ :

$$
\underline{H}(\underline{z}, \bar{z}) \leq H(z, \theta) \leq \bar{H}(\underline{z}, \bar{z}),
$$

and for a given submultiplicative norm $\|\cdot\|$

$$
\begin{aligned}
& \|\bar{H}(\underline{z}, \bar{z})-H(z, \theta)\| \leq \bar{l}_{\bar{H}}\|\bar{z}-z\|+\underline{l}_{\bar{H}}\|\underline{z}-z\|+l_{\bar{H}}, \\
& \|\underline{H}(\underline{z}, \bar{z})-H(z, \theta)\| \leq \bar{l}_{\underline{H}}\|\bar{z}-z\|+\underline{l}_{\underline{H}}\|\underline{z}-z\|+l_{\underline{H}},
\end{aligned}
$$

for some positive constants $\bar{l}_{\bar{H}}, \underline{l}_{\bar{H}}, l_{\bar{H}}, \bar{l}_{\underline{H}}, \underline{l}_{\underline{H}}, l_{\underline{H}}$.

Similar relations for the term $G$ can be also derived using Lemma 7 , i.e. there exist two functions $\bar{G}, G: \mathbb{R}^{2 n+m} \rightarrow$ $\mathbb{R}^{n}$ such that the following inequalities holds for all $u \in \mathcal{U}$, $\theta \in \Theta$ and $\underline{z} \leq z \leq \bar{z}$ :

$$
\underline{G}_{i}\left(\underline{z}, \bar{z}, u_{i}\right) \leq u_{i} B_{i}(z, \theta) z \leq \bar{G}_{i}\left(\underline{z}, \bar{z}, u_{i}\right)
$$

for all $0 \leq i \leq m$, and for a given submultiplicative norm $\|\cdot\|$

$$
\begin{aligned}
& \frac{\left\|\bar{G}_{i}\left(\underline{z}, \bar{z}, u_{i}\right)-G_{i}(z, \theta) u_{i}\right\|}{\left|u_{i}\right|} \leq\left(\bar{l}_{\bar{G}}\|\bar{z}-z\|+\underline{l}_{\bar{G}}\|\underline{z}-z\|+l_{\bar{G}}\right), \\
& \frac{\left\|\underline{G}_{i}\left(\underline{z}, \bar{z}, u_{i}\right)-G_{i}(z, \theta) u_{i}\right\|}{\left|u_{i}\right|} \leq\left(\bar{l}_{\underline{G}}\|\bar{z}-z\|+\underline{l}_{\underline{G}}\|\underline{z}-z\|+l_{\underline{G}}\right),
\end{aligned}
$$

for some positive constants $\bar{l}_{\bar{G}}, \underline{l}_{\bar{G}}, l_{\bar{G}}, \bar{l}_{\underline{G}}, \underline{l}_{\underline{G}}, l_{\underline{G}}$.

\subsection{Interval observer construction}

We are now ready to give the interval observer equations. Let $\bar{z}, \underline{z}$ be the estimates of the transformed state $z$, whose dynamics constitute the interval observer and it is designed as follows:

$$
\begin{aligned}
\dot{\bar{z}}= & A \bar{z}+\bar{G}(\underline{z}, \bar{z}, u)+\bar{F}(\underline{z}, \bar{z}) \\
& +L(y-C \bar{z})+L^{+} \bar{H}(\underline{z}, \bar{z})-L^{-} \underline{H}(\underline{z}, \bar{z}), \\
\dot{\dot{z}}= & A \underline{z}+\underline{G}(\underline{z}, \bar{z}, u)+\underline{F}(\underline{z}, \bar{z}) \\
& +L(y-C \underline{z})+L^{+} \underline{H}(\underline{z}, \bar{z})-L^{-} \bar{H}(\underline{z}, \bar{z}),
\end{aligned}
$$

where the observer gain $L=\left(l_{1}, \ldots, l_{n}\right)^{T}$ has to be designed. Defining the upper error $\bar{e}=\bar{z}-z$ and the lower error $\underline{e}=z-\underline{z}$ their dynamics reads as:

$$
\begin{aligned}
& \frac{d \bar{e}}{d t}=(A-L C) \bar{e}+\bar{\Delta}(\underline{z}, \bar{z}, z, \theta, u, L), \\
& \frac{d \underline{e}}{d t}=(A-L C) \underline{e}+\underline{\Delta}(\underline{z}, \bar{z}, z, \theta, u, L),
\end{aligned}
$$

where $\bar{\Delta}(\underline{z}, \bar{z}, z, \theta, u, L)$ is the sum of the following terms:

$$
\begin{aligned}
\bar{\Delta}_{G}(\underline{z}, \bar{z}, z, \theta, u)= & \bar{G}(\underline{z}, \bar{z}, u)-G(z, \theta) u, \\
\bar{\Delta}_{F}(\underline{z}, \bar{z}, z, \theta)= & \bar{F}(\underline{z}, \bar{z})-F(z, \theta), \\
\bar{\Delta}_{L H}(\underline{z}, \bar{z}, z, \theta, L)= & L^{+} \bar{H}(\underline{z}, \bar{z})-L^{-} \underline{H}(\underline{z}, \bar{z}) \\
& -L H(z, \theta),
\end{aligned}
$$

and $\underline{\Delta}(\underline{z}, \bar{z}, z, \theta, u, L)$ is the sum of

$$
\begin{aligned}
\Delta_{G}(\underline{z}, \bar{z}, z, \theta, u)= & G(z, \theta) u-\underline{G}(\underline{z}, \bar{z}, u), \\
\Delta_{F}(\underline{z}, \bar{z}, z, \theta)= & F(z, \theta)-\underline{F}(\underline{z}, \bar{z}), \\
\Delta_{L H}(\underline{z}, \bar{z}, z, \theta, L)= & L H(z, \theta)-L^{+} \underline{H}(\underline{z}, \bar{z}) \\
& \left.+L^{-} \bar{H}(\underline{z}, \bar{z}) \bar{z}\right) .
\end{aligned}
$$

Corollary 9. For all $z \in \mathcal{Z}, u \in \mathcal{U}$ and $\theta \in \Theta$ there exist positive constants $\underline{l}_{\underline{\Delta}}, \bar{l}_{\underline{\Delta}}, \underline{l}_{\bar{\Delta}}, \bar{l}_{\bar{\Delta}}, l_{\bar{\Delta}}, l_{\underline{\Delta}}$ such that for a chosen submultiplicative norm $\|\cdot\|$

$$
\begin{aligned}
& \|\bar{\Delta}(\cdot, L)\| \leq\left[\bar{l} \overline{\bar{\Delta}}\|\bar{z}-z\|+\underline{l}_{\bar{\Delta}}\|z-\underline{z}\|+l_{\bar{\Delta}}\right](1+\|L\|), \\
& \|\underline{\Delta}(\cdot, L)\| \leq\left[\bar{l}_{\underline{\Delta}}\|\bar{z}-z\|+\underline{l}_{\Delta}\|z-\underline{z}\|+l_{\underline{\Delta}}\right](1+\|L\|) .
\end{aligned}
$$

Lemma 10. Assume that assumptions A1)-A4) are satisfied, then for any $u \in \mathcal{L}_{\infty}$ and any $\left(\bar{e}\left(t_{0}\right), \underline{e}\left(t_{0}\right)\right) \geq 0$ (componentwise), the inequality

holds for every $t \geq t_{0}$.

$$
(\bar{e}(t), \underline{e}(t)) \geq 0
$$

Note that $(\bar{e}(t), \underline{e}(t)) \geq 0$ implies

$$
\underline{z}(t) \leq z(t) \leq \bar{z}(t)
$$

Combination of the last lemma result with a proof of boundedness of the interval observer solutions conclude the consideration.

Theorem 11. Let assumptions A1)-A4) be satisfied and the following matrix inequality be true

$$
D^{T} S+S D+S O^{-1} S+\alpha\|O\| I+Q \preceq 0,
$$

where $D=A-L C$ and $\alpha=3(1+\|L\|)^{2} \max \left\{\bar{l}_{\bar{\Delta}}^{2}+\bar{l}_{\underline{\Delta}}^{2}, \underline{l} \frac{2}{\Delta}+\right.$ $\left.\underline{l}_{\Delta}^{2}\right\}$, for a positive definite and symmetric matrices $\bar{S}, Q, O$ and the constants $\underline{\underline{l}}_{\underline{\Delta}}, \bar{l}_{\underline{\Delta}}, \underline{l}_{\bar{\Delta}}, \bar{l}_{\bar{\Delta}}$ coming from Corollary 9 . Then the variables $\underline{z}(t), \bar{z}(t)$ are bounded and (24) is satisfied for all $t>0$ if it is valid for $t=0$.

Note that if the relation (24) is satisfied and the variables $\underline{z}, \bar{z}$ are bounded, then by standard arguments Jaulin et al. [2001] we can compute $\underline{x}(t)=\underline{\Psi}(\underline{z}(t), \bar{z}(t))$ and 
$\bar{x}(t)=\bar{\Psi}(\underline{z}(t), \bar{z}(t))$ (where $\underline{\Psi}, \bar{\Psi}$ depend on $\Phi_{(10)}$ and $P$ ) such that

$$
\underline{x}(t) \leq x(t) \leq \bar{x}(t)
$$

for all $t \geq 0$, i.e. we obtain the interval estimation for the original nonlinear system (8).

If the output $y$ equals to $h(x)$ and there is no uncertainty $H(x, \theta)$, then clearly the above theorem has more simple conditions, which can be formulated in terms of LMIs for a constructive way of the gain $L$ calculation.

Corollary 12. Let assumptions A1)-A4) be satisfied, $y=$ $h(x)$ in $(8)$ and the following LMI be true for a positive definite and symmetric matrices $S, Q, O$ and $N \in \mathbb{R}^{n}$

$$
\left[\begin{array}{cc}
-O & S \\
S & A^{T} S+S A-C^{T} N^{T}-N C+\alpha\|O\| I+Q
\end{array}\right] \preceq 0
$$

where $\alpha=3 \max \left\{\bar{l}_{\bar{\Delta}}^{2}+\bar{l}_{\Delta}^{2}, \underline{l}_{\Delta}^{2}+\underline{l}_{\Delta}^{2}\right\}$ with the constants $\underline{l}_{\Delta}, \bar{l}_{\Delta}, \underline{l}_{\bar{\Delta}}, \bar{l}_{\bar{\Delta}}$ coming from Corollary 9 . Then for $L=S^{-1} N$ the variables $\underline{z}(t), \bar{z}(t)$ are bounded and (24) is satisfied for all $t \geq 0$.

Thus we designed an interval observer for the uncertain nonlinear system (1)-(2) using the transformation of coordinates calculated for the nominal system (10). The original system may be non-uniformly observable, but if it is possible to extract from (1)-(2) a nominal observable system (10), then the proposed approach establishes the interval observer and the corresponding transformation of coordinates providing the interval state estimation for (1)(2). Let us demonstrate the advantages of this approach on an example of a nonlinear non-observable system.

\section{EXAMPLE}

Consider the following example of the nonlinear system (1)-(2):

$$
\begin{aligned}
\dot{x}_{1} & =x_{2}+a_{1} \sin \left(\theta_{1} x_{1} x_{2}\right), \\
\dot{x}_{2} & =-a_{4} x_{2}-a_{2} \sin \left(\theta_{2} x_{1}\right)+b(y) u, \\
y & =x_{1}+\theta_{3} x_{1} x_{2},
\end{aligned}
$$

where $a_{1}=0.5, a_{2}=0.125, a_{3}=2$ and $a_{4}=1$ are given known parameters, the unknown parameters admit the condition $\left|\theta_{i}\right| \leq \bar{\theta}$ for $i=1,2,3$ with $\bar{\theta}=$ 0.5 . For simulation we will use $\theta_{1}=-0.1, \theta_{2}=-0.2$, $\theta_{3}=0.3[1+0.25 \sin (5 t)+0.125 \cos (8 t)]$ (it is time-varying, representing additional disturbance/noise) and $u(t)=$ $\sin (t)$. We assume that $\left|x_{1}(0)\right| \leq 0.1,\left|x_{2}(0)\right| \leq 0.1$ and that solutions stay bounded and $\left|x_{1}(t)\right| \leq \bar{x}_{1}=0.5$, $\left|x_{2}(t)\right| \leq \bar{x}_{2}=0.1$ Therefore, Assumption A $\left.\overline{4}\right)$ is satisfied. Choose the nominal system

then

$$
f_{1}(x)=x_{2}, f_{2}(x)=-a_{4} x_{2}, h(x)=x_{1},
$$

$$
\begin{gathered}
\delta f_{1}(x, \theta)=a_{1} \sin \left(\theta_{1} x_{1} x_{2}\right), \delta f_{2}(x, \theta)=-a_{2} \sin \left(\theta_{2} x_{1}\right), \\
\delta h(x, \theta)=\theta_{3} x_{1} x_{2} .
\end{gathered}
$$

It is straightforward to check that the nominal system $f$ for the original output $h(x, \theta)$ is not observable, however, the nominal system $(f, h)$ as a linear system in the canonical form is observable. Thus, assumptions A1) and A2) are verified. Assumption A3) is satisfied for the matrix

$$
L=\left[\begin{array}{ll}
1 & 0
\end{array}\right]^{T} \text {. }
$$
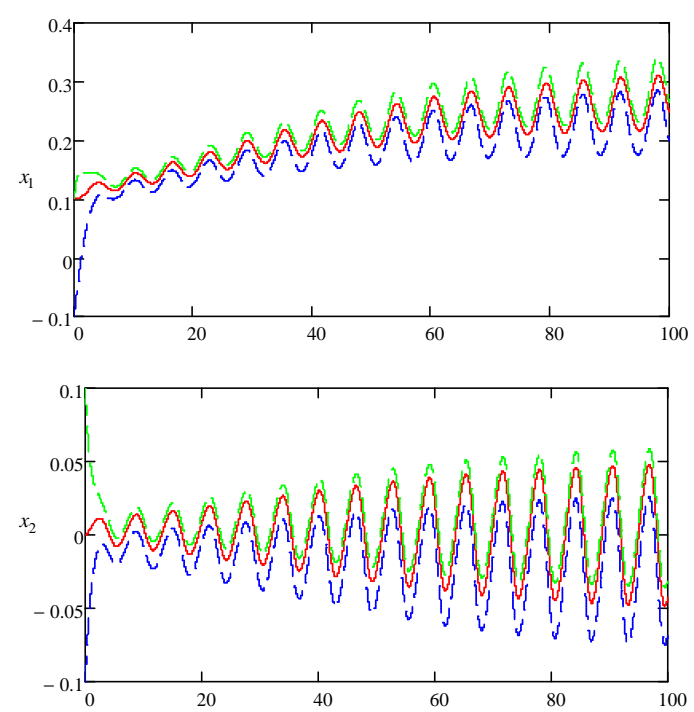

Fig. 1. The results of interval estimation for the coordinates $x_{1}$ and $x_{2}$

Let us compute the bounding functions for $\delta f$ and $\delta h$. Define two functions

$$
\begin{aligned}
\operatorname{Product}(\underline{x}, \bar{x}) & =\left[\begin{array}{l}
\min \left\{\bar{x}_{1} \bar{x}_{2}, \underline{x}_{1} \bar{x}_{2}, \bar{x}_{1} \underline{x}_{2}, \underline{x}_{1} \underline{x}_{2}\right\} \\
\max \left\{\bar{x}_{1} \bar{x}_{2}, \underline{x}_{1} \bar{x}_{2}, \bar{x}_{1} \underline{x}_{2}, \underline{x}_{1} \underline{x}_{2}\right\}
\end{array}\right], \\
{\left[\begin{array}{l}
\underline{\sin }(\underline{x}, \bar{x}) \\
\overline{\sin }(\underline{x}, \bar{x})
\end{array}\right] } & =\left[\begin{array}{l}
\sin (\underline{x}) \\
\sin (\bar{x})
\end{array}\right]
\end{aligned}
$$

corresponding to the interval of the product $x_{1} x_{2}$ for $x=\left[\begin{array}{ll}x_{1} & x_{2}\end{array}\right]^{T}$ with $\underline{x} \leq x \leq \bar{x}$ and the interval of the function $\sin (x)$ for a scalar $x$ with $\underline{x} \leq x \leq \bar{x}$ (for $|x| \leq \pi / 2)$. Then

$$
\begin{aligned}
\frac{\delta f_{1}}{\bar{\delta}_{1}}(\underline{x}, \bar{x}) & =a_{1} \underline{\sin }\{\varrho(\bar{\theta}, \underline{x}, \bar{x})\}, \\
\overline{f f}_{1}(\underline{x}, \bar{x}) & =a_{1} \overline{\overline{\sin }}\{\varrho(\bar{\theta}, \underline{x}, \bar{x})\}, \\
\frac{\delta f_{2}}{2}(\underline{x}, \bar{x}) & =-a_{2} \overline{\sin }\left\{\operatorname{Product}\left(\left[\begin{array}{c}
-\bar{\theta} \\
\bar{\theta}
\end{array}\right],\left[\begin{array}{c}
\bar{x}_{1} \\
\bar{x}_{1}
\end{array}\right]\right)\right\}, \\
\overline{\delta f}_{2}(\underline{x}, \bar{x}) & =-a_{2} \underline{\sin }\left\{\operatorname{Product}\left(\left[\begin{array}{c}
-\bar{\theta} \\
\bar{\theta}
\end{array}\right],\left[\begin{array}{c}
\bar{x}_{1} \\
\bar{x}_{1}
\end{array}\right]\right)\right\}, \\
{\left[\begin{array}{c}
\delta h_{1}(\underline{x}, \bar{x}) \\
\overline{\delta h}_{1}(\underline{x}, \bar{x})
\end{array}\right] } & =\varrho(\bar{\theta}, \underline{x}, \bar{x}),
\end{aligned}
$$

where

$$
\varrho(\bar{\theta}, \underline{x}, \bar{x})=\operatorname{Product}\left(\left[\begin{array}{c}
-\bar{\theta} \\
\bar{\theta}
\end{array}\right], \operatorname{Product}(\underline{x}, \bar{x})\right) .
$$

Take

$$
\bar{l}_{\underline{\Delta}}=\bar{l}_{\bar{\Delta}}=\underline{l}_{\bar{\Delta}}=\underline{l}_{\underline{\Delta}}=\left[a_{2}+\left(a_{1}+1\right) \bar{x}_{2}\right] \bar{\theta},
$$

then $\alpha=3(1+\|L\|)^{2} \max \left\{\bar{l}_{\bar{\Delta}}^{2}+\bar{l}_{\underline{\Delta}}^{2}, \underline{l}_{\bar{\Delta}}^{2}+\underline{l}_{\Delta}^{2}\right\}=0.227$. For the chosen parameters, the matrix inequality from Theorem 11 is satisfied for:

$$
S=\left[\begin{array}{cc}
0.5 & -0.4 \\
-0.4 & 1
\end{array}\right], O=I, Q=0.01 I,
$$

thus all conditions of Theorem 11 have been verified. The results of the interval estimation are given in Fig. 1. 


\section{CONCLUSION}

The problem of state estimation is studied for an uncertain nonlinear system not in a canonical form. The uncertainty is presented by a vector of unknown time-varying parameters, the system equations depend on this vector in a nonlinear fashion. It is assumed that the values of this vector of unknown parameters belong to some known compact set. The idea of the proposed approach is to extract a known nominal observable subsystem from the plant equations, next a transformation of coordinates developed for the nominal system is applied to the original one. The interval observer is designed for the system transformed to a canonical form. It is shown that the residual nonlinear terms dependent on the vector of unknown parameters have linear upper and lower functional bounds, that simplifies the interval observer design and stability/cooperativity analysis.

\section{REFERENCES}

G. Besançon, "Nonlinear observers and applications", Vol 363, Lecture Notes in Control and Information Science, Springer Verlag, Berlin, 2007.

G. Bitsoris, "Principle de Comparaison et Stabilité des Systèmes Complexes", Ph.D Thesis, Paul Sabatier University of Toulouse, France, No. 818, 1978.

M. Dambrine, "Contribution l'étude des systèmes à retards", Ph.D. Thesis, University of Sciences and Technology of Lille, France, No. 1386, 1994.

M. Dambrine, A. Goubet, J. P. Richard, "New results on constrained stabilizing control of time-delay systems", Proc. 34th IEEE CDC, New Orleans, pp. 2052-2057, 1995.

M. Dambrine, J. P. Richard, "Stability Analysis of TimeDelay Systems", Dynamic Systems and Applications, Vol. 2, 1993, pp. 405-414.

M. Dambrine, J. P. Richard, "Stability and Stability Domains Analysis for Non-linear Differential-Difference Equations ", Dyn. Syst. and Applications, Vol. 3, 1994, pp. $369-378$.

J. Gauthier, H. Hammouri, and S. Othman, "A simple observer for nonlinear systems: Applications to bioreactors," IEEE Trans. Automat. Contr., vol. 37 no. 6, 1992, pp. $875-880$.

J.-L. Gouzé, A. Rapaport, Z. Hadj-Sadok, "Interval observers for uncertain biological systems", Ecological Model., 133, 2000, pp. 45-56.

Lj. T. Grujic̀, A.A. Martynyuk,M. Ribbens-Pavella, "Large Scale Systems Stability under Structural Perturbations", Lecture Notes in Control and Information Sciences, Vol.92, Springer Verlag, New York 1987.

L. Jaulin, M. Kieffer, O. Didrit, E. Walter, "Applied Interval Analysis", Berlin: Springer, 2001.

E. Kamke, "Zur Theorieder Systemegewohnlicher Dierentialgliechungen II", Acta Math. 58, 1932, pp. 57-85.

V. Laksmikantham and S. Leela, "Differential and integral inequalities, Vol I and II", Academic Press, New York, 1969.

V.M. Matrosov, "Vector Lyapunov Functions in the Analysis of Nonlinear Interconnected System", Symp. Math. Academic Press, New York, Vol.6, 1971, pp.209-242.

F. Mazenc, O. Bernard, "Asymptotically Stable Interval Observers for Planar Systems With Complex Poles",
IEEE Trans. Autom. Control, Vol. 55 N 2, 2010, pp. $523-527$.

F. Mazenc, O. Bernard, "Construction of ISS Interval Observers for Triangular Systems", Proc. 51st IEEE Conference on Decision and Control, Maui, pp. 6780$6785,2012$.

N. Meslem, N. Ramdani, "Interval observer design based on nonlinear hybridization and practical stability analysis", Int. J. Adapt. Control Signal Process., Vol. 25, 2011, pp. 228-248.

M. Müller, "Über das fundamental theorem in der theorie der gewöhnlichen differentialgleichungen", Math. Z, Vol. 26, 1926, pp. 619-645.

H. Nijmeijer, T.I. Fossen, "New Directions in Nonlinear Observer Design”, Springer-Verlag, London, U.K., 1999.

W. Perruquetti, J.P. Richard, P. Borne, "Vector Lyapunov functions: recent developments for stability, robustness, practical stability and constrained control", Nonlinear Times 65 Digest, Vol. 2, 1995, pp. 227-258.

W. Perruquetti, J.P. Richard, LJ. T. Grujić, P. Borne, "On Pratical Stability with the Settling Time via Vector Norms", Int. J. Control, Vol. 62 No.1, 1995, pp.173-189.

T. Raïssi, D. Efimov, A. Zolghadri, "Interval state estimation for a class of nonlinear systems", IEEE Trans. Automatic Control, 57, 2012, pp. 260-265.

H.L. Smith, "Monotone Dynamical Systems: An Introduction to the Theory of Competitive and Cooperative Systems", vol. 41 of Surveys and Monographs, AMS, Providence, 1995.

H. Tokumaru, N. Adachi, T. Amemiya, "Macroscopic stability of interconnected systems", Proc. of IFAC 6th World Congress, Boston, Paper 44.4, 1975.

E. Walter, J. Norton, H. Piet-Lahanier, M. Milanese, "Bounding Approaches to System Identification", Perseus Publishing, 1996.

T. Wazewski, "Systèmes des Équations et des Inégalités Différentielles Ordinaires aux Seconds Membres Monotones et leurs Applications", Ann. Soc. Polon. Math., Vol. 23, 1950, pp.112-166. 